\title{
Optimization of the Die Topology in Extrusion Processes
}

\author{
T. Kloppenborg ${ }^{1, a}$, M. Schikorra ${ }^{1, b}$, J. P. Rottberg ${ }^{1, c}$, and A. E. Tekkaya ${ }^{1, d}$ \\ ${ }^{1}$ Institute of Forming Technology and Lightweight Construction, Technische Universität Dortmund, \\ Baroper Str. 301, Dortmund 44227, Germany \\ aThomas.Kloppenborg@iul.uni-dortmund.de, ${ }^{\mathrm{b}}$ Marco.Schikorra@iul.uni-dortmund.de, \\ 'Jan.Rottberg@iul.uni-dortmund, 'Erman.Tekkaya@iul.uni-dortmund.de
}

Keywords: Extrusion, topology, optimization

\begin{abstract}
This paper presents the results of investigations on topology optimizations in extrusion dies. The change of material viscosity of finite elements in the numerical model is utilized to allow or to block the material flow through the finite elements in simplified two-dimensional extrusion models. Two different optimization procedures are presented. In the first part of the paper dead zones in a flat and in a porthole die were improved by enhance the streamlining of the extrusion die. In the second part an evolutionary optimization algorithm has been used to optimize the extrusion die topology in order to reduce the difference between the strand exit velocities in a multi extrusion process. Finally, both methods were sequentially combined.
\end{abstract}

\section{Introduction}

The commercial extrusion process is an important manufacturing method to produce profiles used in different applications, for example automotive and aerospace engineering. In recent decades especially the extrusion of materials with low density, such as aluminum or magnesium, became more interesting for the industry to reduce the weight of the components. Typical examples are car space-frames and similar structures where the main supporting elements are utilized for the stabilization of the entire structure. These components are often manufactured by extrusion; this is why extrusion is a successful application in lightweight structures.

Die manufacturing for complex profiles is mainly based on expert knowledge in design, manufacture, and adjustment, which has been gathered over many decades and is mostly kept confidential by the manufacturing companies. Hence, the manufacturing process requires time-and cost-consuming trial-and-error experiments to achieve an optimized die design. In 1988, Akeret noted that die design and die correction is more an art than a science-based technology. The lack of quantitative models and rules regarding the control of the metal flow were a serious obstacle for a computer-aided design [1].

In recent decades the extrusion process simulation has been increasingly used to analyze the conditions which are not measurable, for example distribution of temperature, pressure, or material flow in complex and/or porthole dies [2, 3]. Hence, as a result of the revised numerical codes [4] the extrusion process simulation is just before being introduced in extrusion companies.

Research and development centres like software developers and universities currently take a step further than the simple simulation of the process. They realized that the responses of the numerical calculations can be used for process optimization or, in fact, for process adjustment by optimization algorithms. Ongoing projects, for example, use parametric optimization for a defined control of the material flow by adaptation of the bearing geometry $[5,6]$. Other works use a multi-objective optimization to improve the deviation of the effective strain as a measurement for the material quality, combined with exiting velocity optimization [7].

Contrary to previously published works on extrusion die optimization, this paper will focus on the simulation-based optimization of the die topology. A first approach is used to homogenize the 
material flow in the die by improving the die geometry. Especially dead zones in the material flow, which occur in areas of acute angles or behind undercuts, have been reduced by a adjustment of the die streamlining. But the application in a multi-extrusion process leads to different exit velocities. Therefore, in a second approach the application of an evolutionary algorithm is presented, which was defined and developed for an automated free topology optimization of the extrusion die. The evolutionary algorithms are based on the idea of copying the Darwin-Evolution-Model. The evolutionary operations mutation, recombination and production have been used to generate equal strand exit velocities in a multi-extrusion process with a porthole die in a simplified twodimensional model.

\section{Model Setup}

Mesh Generation. There are two methods for mesh generation, Solid-Modeling and DirectGeneration-Method in the used ANSYS software. Solid-Modeling creates a mesh on a solid geometry, implying the advantage that designs generated in CAD programs can be used to automesh the model. For this type of meshing, a meshing tool is needed which provides the needed discretization for the analyzed problem. The Direct-Generation-Method generates nodes and elements and thus, the mesh itself. The method requires knowledge about the model geometry and programming knowledge. The Direct-Generation-Method has been used to create the extrusion model. To simplify the meshing process and to reduce calculation time only two-dimensional models were considered (Fig. 1). They consist of four node quad elements with linear shape functions. To simulate a multi-extrusion process with a porthole die, a barrier in the center of the material flow before the die orifice was considered in a second model shown on the right front side of Fig. 1.

\section{Model - unmeshed flat die}
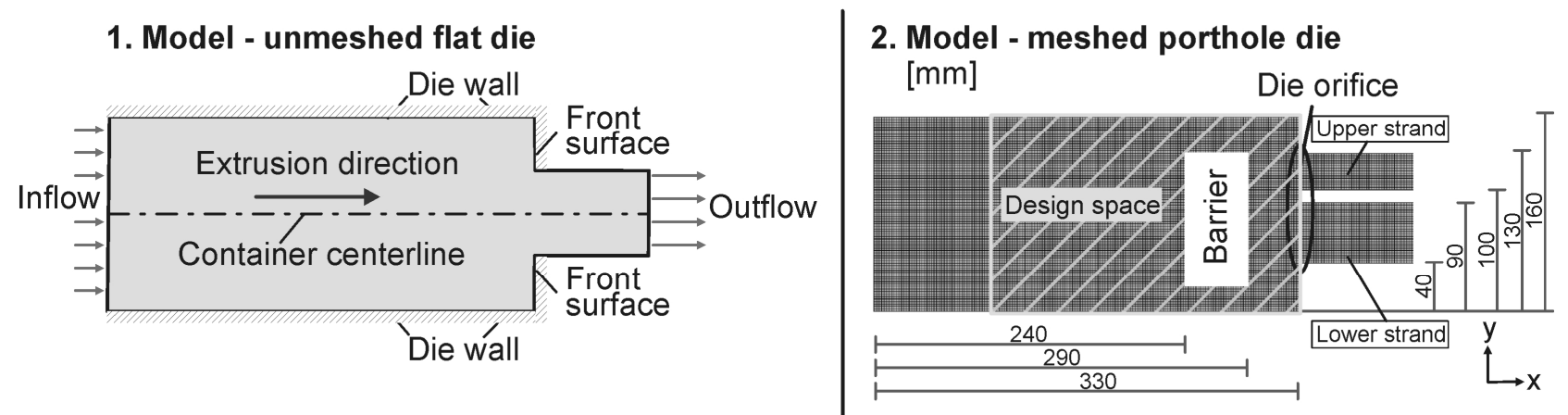

Figure 1: Geometry and FE-model used for the optimization processes

Material. The element type FLUID 141 was used for the extruded material, which is implemented in the ANSYS software. The extrusion has been adapted to the mechanical properties of $450^{\circ} \mathrm{C}$ preheated aluminum EN AW-6060 (Material-1) by adjusting the viscosity by means of achieving a die pressure of $200 \mathrm{MPa}$. Here, a viscosity of $10 \mathrm{~kg} / \mathrm{mms}$ and a density of $2.7 \mathrm{e}-09 \mathrm{~kg} / \mathrm{mm}^{3}$ were applied to simulate the extrusion of aluminum. During optimization another material (Material-2) was defined which represents die material with an extremely high density and viscosity to prevent material flow. Preheating conditions, heat exchange and the thermal evolution were not taken into account.

Boundary Conditions. The nodes on the left border of the models are assigned to the inflow velocity representing the stamp and the extrusion direction. The border nodes of the top side and bottom side of the strands as well as the nodes on the die wall are assigned with a velocity of zero normal to the extrusion direction. Thus, the nodes of the die front surface are assigned with a velocity of zero in the extrusion direction, representing the front surface. No pressure is assigned to the nodes on the outflow. In the second model the nodes around the barrier are assigned with a velocity of zero to avoid material flow through them, analogous to a porthole die. 


\section{Manual Improvement of Dead Zones}

During the extrusion process the preheated material is pressed through a die to form the profile cross section. Depending on the complexity of the die geometry, areas of different flow velocity occur. The distribution is mainly physical-based by friction or sticking effects in the contact zone between extrusion material and die wall. Hence, there emerge areas where the material stops flowing, especially in acute angles or behind undercuts. These areas are called dead zones. In Fig. 2, the dead zones are shown for the initial design of the multi-extrusion process model.

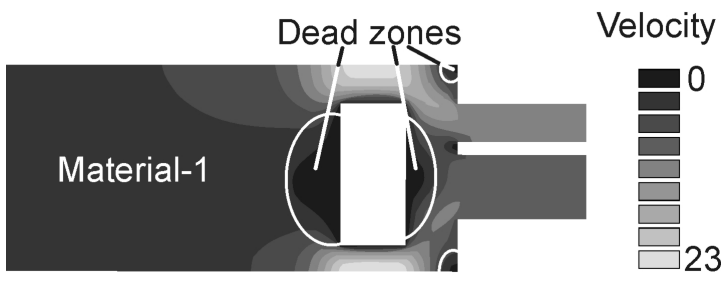

Figure 2: Example of dead zones in the material flow for the initial die design

Manual Improvement Procedure. Due to the problems which are caused by the appearance of dead zones in extrusion dies an optimization of the material flow has been carried out. The optimization is mainly focused on the method of the topology optimization, friction effects were unaccounted. The objective of the optimization is to reduce the dead zones in the material flow. First, an initial numerical calculation with Material-1 in all elements is performed (Fig. 2). The material flow velocity in each element was identified to sort them according to value. Then the elements with less than $10 \%$ of the maximum flow velocity were selected and assigned to Material2. The extremely high viscosity of this material leads to a blocking of the material flow through these elements in the next calculation step (Fig. 3). The restart for the numerical calculation can be done manually.

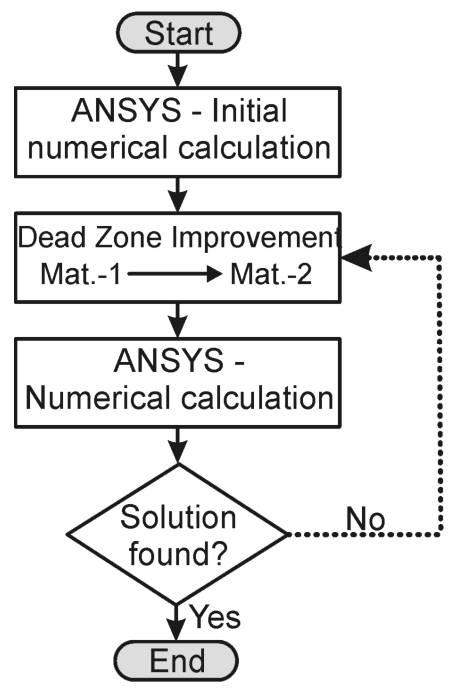

Figure 3: Process procedure to improve dead zones

Results for the Flat Die. In a first calculation run a simplified extrusion model with a flat die was analyzed and than sequentially improved. The initial step was performed to identify the dead zones in the numerical model. As shown in Fig. 4, the dead zones occur in the upper and lower corner of the front surface. In optimization step 1 it can be seen that the material in the initial step shears along the corners at an angle of approximately $40^{\circ}$. The elements which have been changed from Material-1 to Material-2 are not considered further due to the low flow velocity. The angle increased in further steps due to the change in material flow conditions. The geometry of the die gets more streamlined during the improvement of the dead zones, but an ideal improved model without dead 
zones could not be achieved. Hence, the uneven line along the element edges, which were changed in material, implements local dead zones in the changed models.
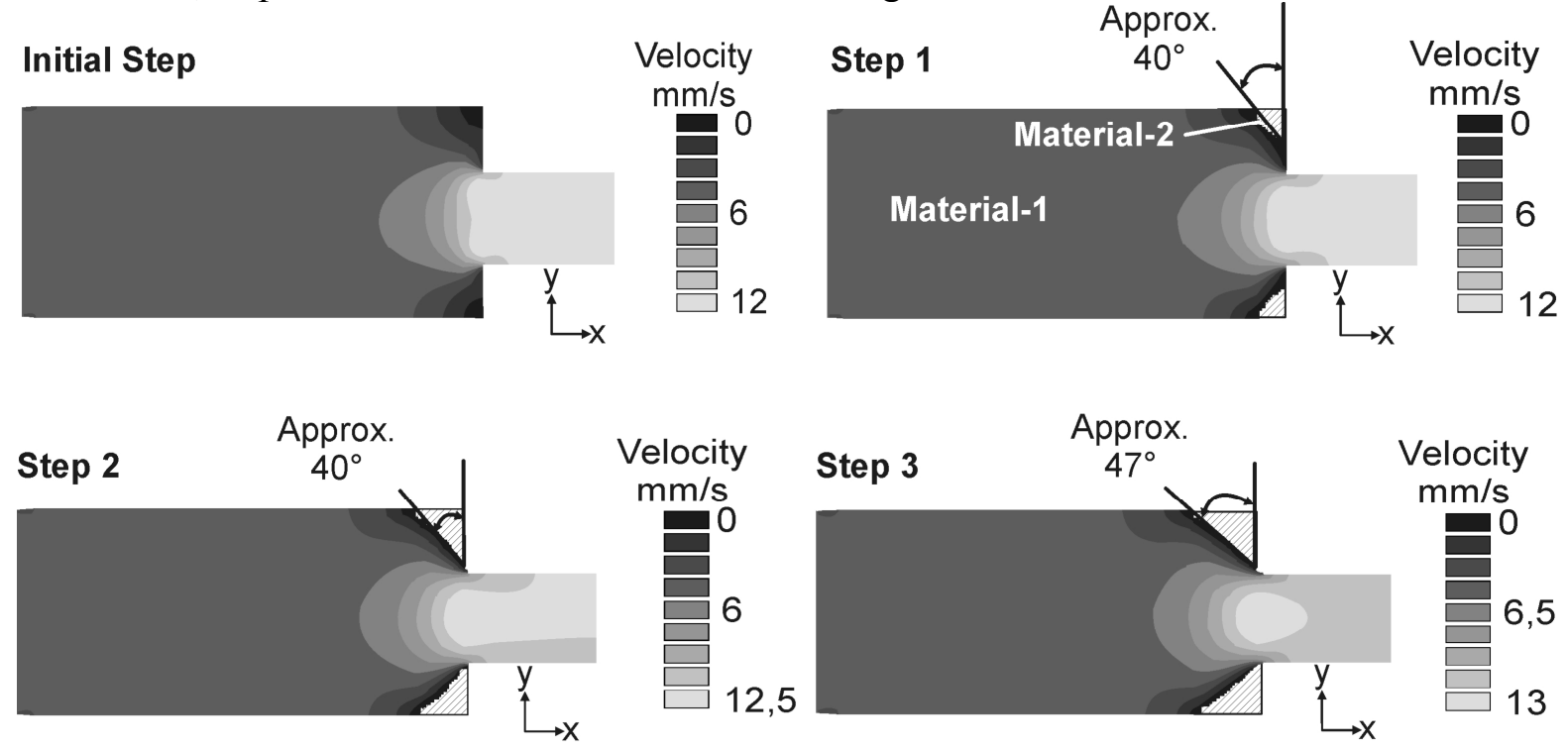

Figure 4: Improvement steps in a flat die to avoid dead zones

The shear angles of the manual improvement steps of the simplified finite element model are approximately concordant with experimental results of Hinkfort [8], as it can be seen in Fig. 5 .

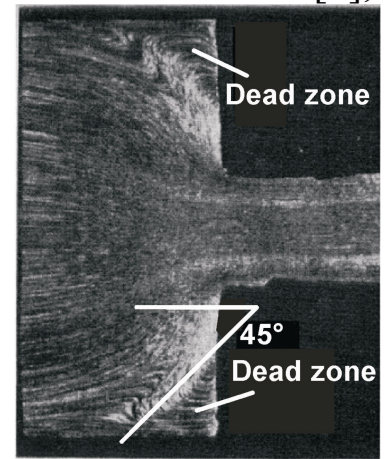

Figure 5: Position and angle of dead zones [8]

Results for the Porthole Die. To raise the extrusion model complexity, the dead zones of a porthole die were improved by four manual optimization steps. Within the loops between numerical calculation and improvement step the number of elements, which were blocked for flow reasons, increases (Fig 4). It is obvious that the barrier geometry changes during the optimization steps to an streamline-shaped geometry. In step 4, the design space limits the change of the barrier geometry, thus on the left hand side no more elements are changed in material. The dead zones in the corners of the front surface are optimized during step 1, 2, 3 and 4. In this area the die geometry is chamfered analogue to the results of the flat die. In step 4 it is shown that the material flow is divided into two different flows after passing the barrier. Furthermore, the barrier changes from the mostly symmetric shape in step 1 and step 2 to an asymmetric shape in step 3 and step 4. 

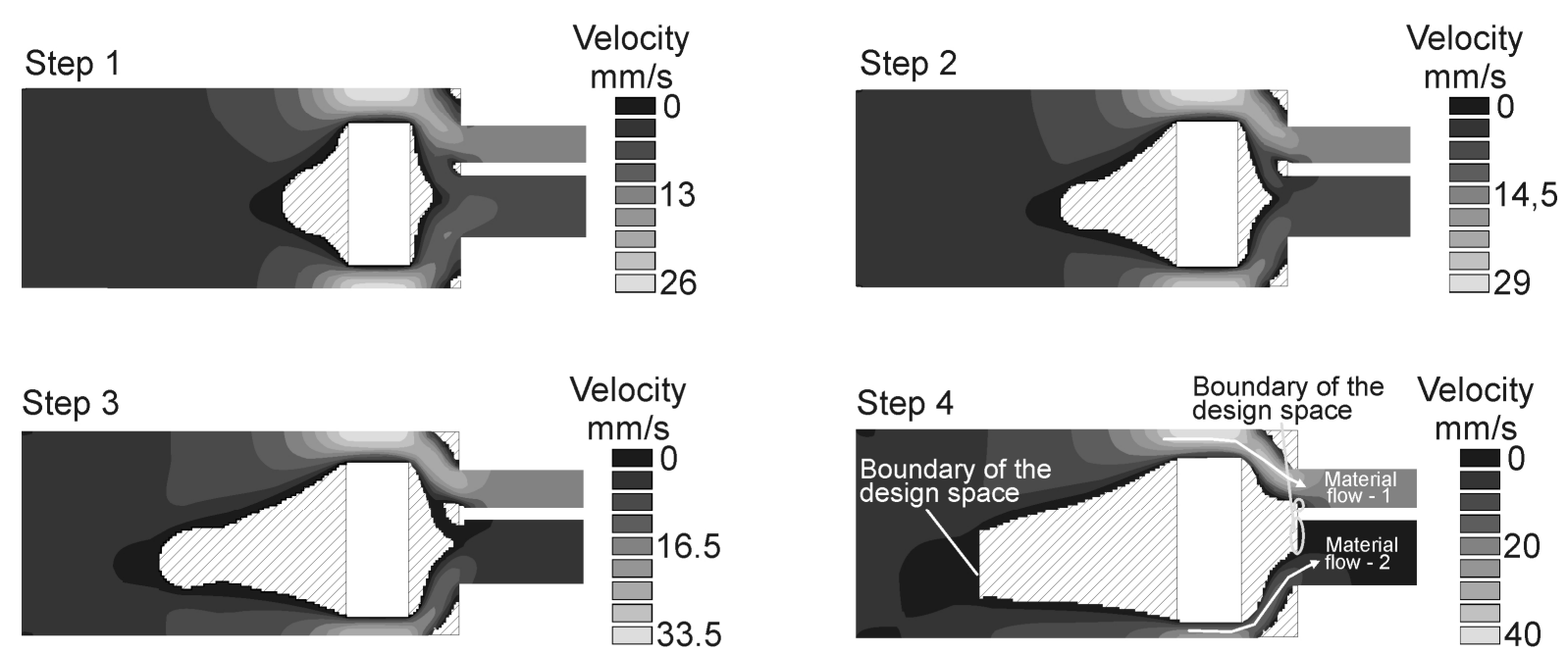

Figure 6: Improvement steps in a porthole die to avoid dead zones

The difference between the profile exit velocities increases with each improvement step. This can lead to production problems. Therefore a genetic algorithm is introduced in the second approach described in the next section.

\section{Optimization of the Material Flow by Reducing the Differences in Strand Exit Velocity}

For handling reasons and for subsequent processing it can be necessary that the manufactured profiles of the multi-extrusion process have been extruded with the same length. The difference in profile length is the result of different strand exit speeds during the multi-extrusion process. The adjustment of the die orifice, the individual position to the container centerline, and the different profile cross sections have, for example, an influence on the material flow, which can induce a difference between the strand exit speeds. Thus, a temperature gradient in the die can have an effect on the flow stress and, thereby, on the material flow in the feeders. The problems occur not only in multi-extrusion processes, but also during the extrusion of complex hollow cross sections if the profile thickness is different along the profile center line $[5,9]$; here, for example unwanted bending or torsion effects can occur.

In practice, these problems are eliminated by the use of expert knowledge in design and manufacturing and by manual adjustment of the die. In order to monitor the influence of the adjustments, time consuming and costly trial-and-error experiments are necessary. In contrast to this, a simulation-based optimization of the exit velocities in the orifice of the extrusion die is presented in the following. The objective of the optimization is to achieve equal exit velocities in the strands of the multi-extrusion process model. Before starting the automated optimization the design space, the design variables, the termination criterions, and the optimization algorithm were defined.

Design Space and Design Variables. The design space is defined by the finite elements between the die orifice and the inflow of the material as shown in Fig. 2. The elements which are assigned to Material-1 and which are at the boundary of the die wall or in contact with Material-2 are defined as design variables for the next optimization run. The type of design variable is discrete; it can only be changed from Material-1 to Material-2.

Fitness Function and Termination Criterion. The fitness of the individuals " $i$ " is evaluated by the difference of the averaged exit velocities at the nodes in the upper strand $\left(v_{\text {upper }}(i)\right)$ and the lower strand $\left(v_{\text {lower }}(i)\right)$. The objective of the optimization problem is to minimize the result of the fitness function. For a fitness value less than $0.1 \mathrm{~mm} / \mathrm{s}$ the termination criterion is reached.

$$
\text { fitness }(i)=\left|v_{\text {upper }}(i)-v_{\text {lower }}(i)\right|
$$


Genetic Algorithm. Genetic algorithms are part of the evolutionary algorithms with the idea of implement a Darwin-Evolution-Model approach for process optimization. To achieve an equal exit velocity in both extrusion strands, the evolution operations mutation, recombination, and production have been used. For an individual of the start population, elements along the die wall of the multiextrusion process model are stochastically selected. For each selected element the immediate neighbor elements are selected additionally by a random number between 1 and 4 to produce noses along the die wall with different depths (Fig. 7) to change the material flow. All selected elements are assigned to Material-2.
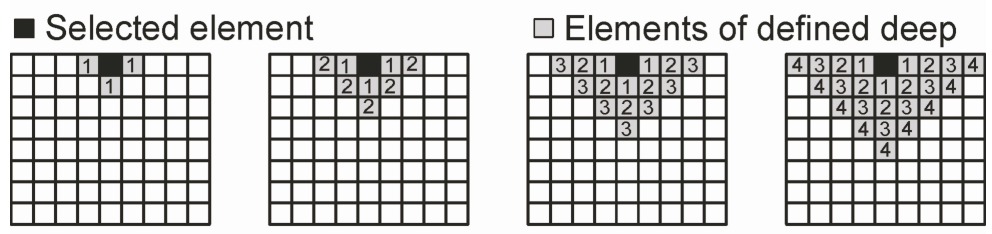

Figure 7: Definition to create noses in the material flow

After creating the initial generation with a population of 10 individuals, the velocities in the strands are evaluated. Within the optimization the fitness value for each individual has been calculated to sort them according fitness. Next, the individuals are utilized for the classical genetic algorithm, using the genetic operations to find the children of the parent generation. The operations are sequentially applied on the 10 individuals.

The algorithm runs as long as the population has not reached start population value (Fig. 8a). The classic genetic algorithm uses a random number between 0 and 1 to decide which operation should be conducted to find new individuals for the next generation. If the number is less than 0.6, a recombination of two parents is used. Thereby the geometry of the best two individuals is horizontally divided and then one half of both individuals is exchanged (Fig. 8c, recombination). If the random number is between 0.6 and 0.7 , a mutation of the next individual is utilized. Thereby, a stochastic selection is used for a mutation of each nose of one individual by extending, reducing, or keeping the depth (Fig. 8c, mutation). The operation production is utilized when the number is larger than 0.7 . The genetic operation production finds a new independent individual, as described for the start population. The probabilities to use recombination, mutation, or production have been taken from literature [10].

(a) Programm procedure

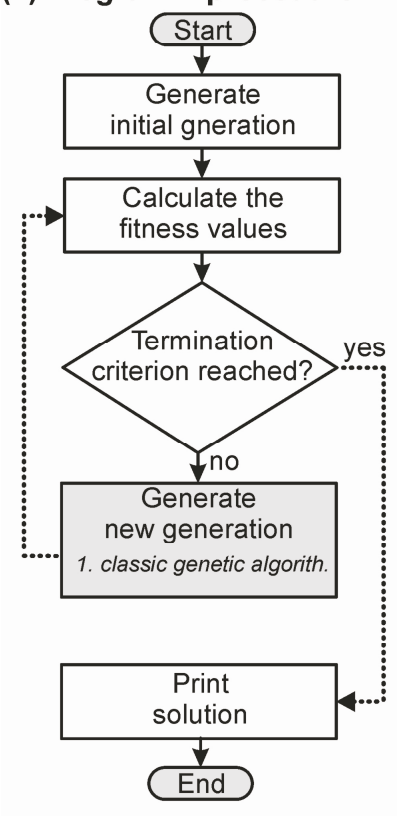

(b) Classic genetic algorithm

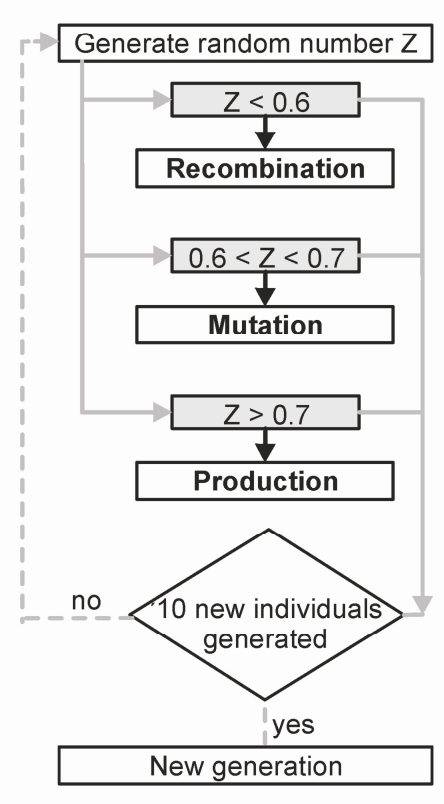

(c) Genetic operations

1. Recombination

Individual 1 Individual 2

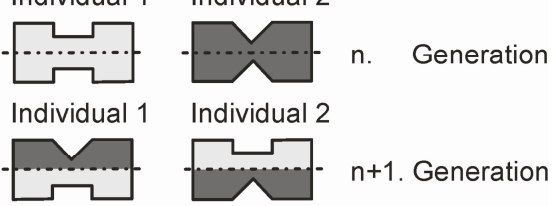

2. Mutation

Individual

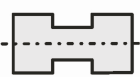

n. Generation

Individual

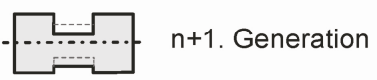

3. Production

New individual

$n+1$. Generation

Figure 8: (a) Optimization program procedure; (b) Used classic genetic algorithm;

(c) Examples for genetic operations 
Results. The evolutionary algorithm is not deterministic. Due to this, it is possible to find different results for the same starting conditions. To study this, seven optimizations were conducted. The number of generations in every optimization run is representative for the convergence of the algorithm. In average, 9 generations with 10 individuals each, were needed to optimize the strand exit velocities. The scatter in the needed generations is extremely high between 3 generations in the second run and 15 generations in the seventh run.

To compare the different extrusion die topologies, the optimized geometries of the first four optimization runs are presented in Fig. 9. It is obvious that the changes in geometry are very different to each other. As presented in Fig. 2, the upper strand velocity was higher than the lower strand velocity before optimization. In the optimized geometry of run 1 , run 3 , and run 4 the material flow in the upper feeder slows down due to the geometry change between barrier and container wall (A). The material flow is changed in the upper feeder, thus, an equal exit velocity in both strands occurs. In contrast to this, in run 2 the maximum velocity is $26 \mathrm{~mm} / \mathrm{s}$, which is much smaller than in the other results. Very small variations at the barrier and on the lower side of the container wall cause equal exit velocities. It can also be seen that in run 1, different from the other runs, material from the upper feeder now flows towards the lower strand.
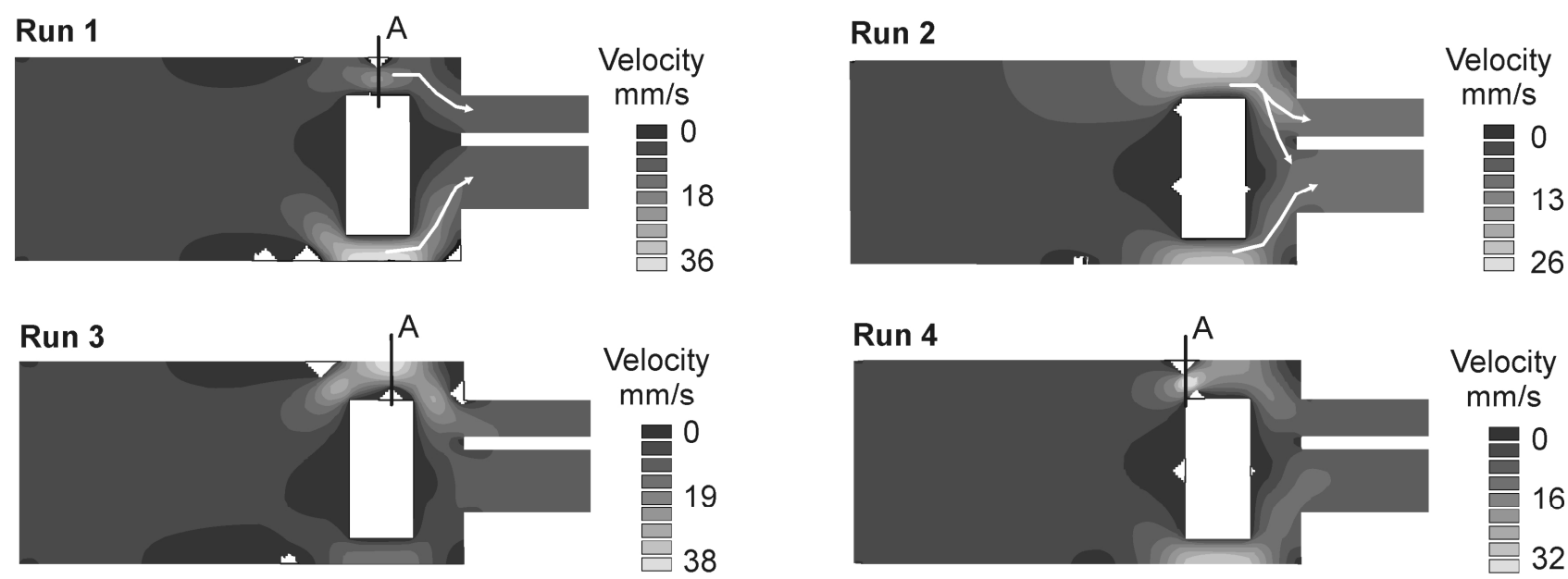

Figure 9: Optimized extrusion die for different optimization runs

\section{Consecutively Combination of the Approaches}

In the previous parts the working principle of the topology optimization methods for optimizing extrusion dies were demonstrated. Finally, the algorithm intended to improve dead zones in the extrusion die and the algorithm intended to achieve equal strand exit velocities were combined. On the one hand, they can be used consecutively, for example by reducing the dead zones first and achieving the equal strand exit velocity afterwards. On the other hand, it may also be beneficial to use both optimization procedures alternately. This means that every new generation of individuals, which causes equal strand exit velocities, is improved by a minimization of the dead zones. In the following, the results are demonstrated exemplarily by optimizing the dead zones first and subsequently achieving equal strand exit velocities.

Two manual optimization steps were applied to reduce the dead zones before the automated genetic algorithm was utilized to achieve equal strand exit velocities. It can be seen that due to the reduced cross section of the feeders the maximum velocity increases up to $56 \mathrm{~mm} / \mathrm{s}$. The maximum velocity occurs in both runs in the lower feeder (Fig. 10). 


\section{Run 1}

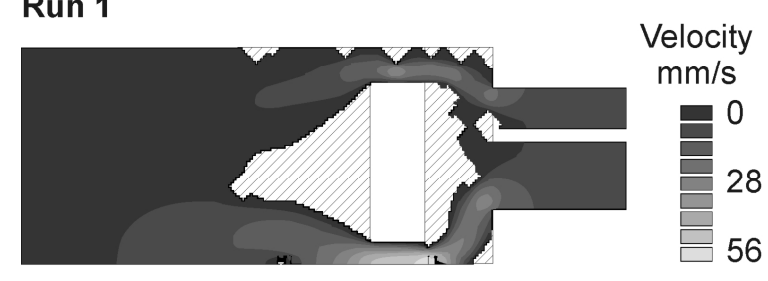

Run 2

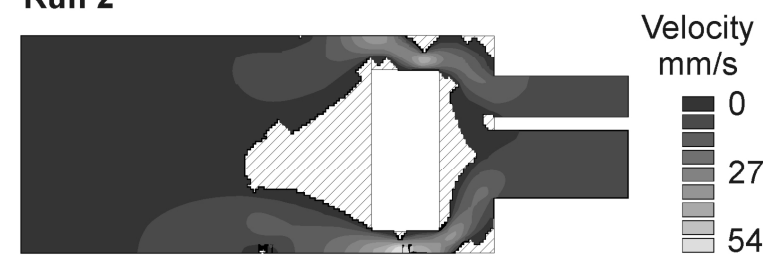

Figure 10: Results using a sequentially combined optimization

\section{Conclusion}

Methods for the optimization of dead zones and strand exit speeds by topology optimization of two extrusion dies are presented, analyzed and optimized using these methods individually or consecutively. The optimization method developed to avoid dead zones accounts for streamlined die geometries in the application of a flat and a porthole die on simplified two-dimensional extrusion models. The genetic algorithm to achieve equal strand exit velocities requires in average nine generations with 10 individuals for the topology optimization. Fore these simplified models every calculation has been done in a short time, but it has to be taken into account that for more complex geometries and three-dimensional models the calculation time can strongly. Therefore, aspects of parallelization of calculation will be utilized in the future.

\section{Acknowledgement}

This paper is based on investigations within scope of the Transregional Collaborative Research Center/ TR10 and is kindly supported by the German Research Foundation (DFG).

\section{References}

[1] R. Akeret, W. Strehmel: Control of Metal Flow in Extrusion Dies, Proceedings of the 4th International Aluminum Extrusion Technology Seminar (1988), Chicago, USA, pp. 357-367

[2] M. Schikorra, M. Kleiner: Simulation-Based Analysis of Composite Extrusion Processes, In: Vol. 56/1 CIRP Annals (2007), General Assembly, Dresden, Germany, pp. 317-320

[3] M. Schikorra, L. Donati, L. Tomesani, M. Kleiner: Role of Friction in Extrusion of 6060, Journal of Materials Processing Technology, Volume 191, Issues 1-3, pp. 288-292

[4] N.N.: Results of the Extrusion Benchmark 2007, Proc. of the $2^{\text {nd }}$ Extrusion Benchmark (2007), ISBN 978-88-548-1286-4, Bologna, Italy

[5] M. P. Reddy: Analysis and Design Optimization of Aluminum Extrusion Dies, Proceedings of the $8^{\text {th }}$ Aluminum Extrusion Technology Seminar (2004), Orlando, USA, Vol. 2, pp.171-178

[6] T. Kloppenborg, M. Schikorra, M. Schomäcker, A. E. Tekkaya: Numerical Optimization of Bearing Length in Composite Extrusion Processes, Proc. of the $2^{\text {nd }}$ Extrusion Benchmark (2007), ISBN 978-88-548-1286-4, Bologna, Italy

[7] D. Y. Yang: Computer-Aided Optimization of Metal Forming Processes as Applied to 3-D Extrusion of Profiles, Proceedings of the $8^{\text {th }}$ ICTP (2005), Verona, Italy

[8] P. Hinkfoth: Massivumformung, 1. Edition, ISBN 978-3-86130-184-4, Verlagshaus MainzAachen, Germany (2003)

[9] X. Zhang, J. Heathcock: Modeling of Metal Flow for Bearing Design, Proceedings of the $7^{\text {th }}$ International Aluminium Extrusion Technology Seminar (2000), pp. 169-176

[10]W. Kinnebrock: Optimierung mit genetischen und selektiven Algorithmen, ISBN-10: 3486226975, ISBN-13: 978-3486226973, Oldenbourg Publisher (1994) 